\title{
Recursos educativos digitales propuestos por el Ministerio de Educación de Chile para atender a estudiantes que asocian algún tipo de discapacidad
}

Yorka Tatiana Ortiz Ruiz1

Olivia del Carmen Soto Jipoulou ${ }^{2}$

Sonia Isabel Muñoz Muñoz

Recepción: 25-04-2018 / Aceptación: 03-07-2018

\section{Resumen}

Los recursos educativos digitales contribuyen en forma indiscutible a la construcción de comunidades educativas inclusivas, contribuyendo con ambientes de aprendizaje favorables en los estudiantes a partir de la interacción entre ambos, ya que respeta los ritmos y estilos de aprendizaje, aportando a la innovación y orientada a la igualdad. El objetivo de esta investigación fue analizar los recursos educativos digitales propuestos por el Ministerio de Educación de Chile para estudiantes que asocian algún tipo de discapacidad, desde una perspectiva mixta, en los ámbitos técnicos, pedagógicos y de accesibilidad. Los resultados dejan en evidencia falencias básicas como que los recursos no están disponibles, presentan debilidades pedagógicas; se concentran mayoritariamente en necesidades educativas de tipo permanente, la mayor parte son recursos extranjeros y lo más importante es que no se cuenta con recursos educativos clasificados a nivel de excelencia.

Palabras clave: Educación, tecnologías, recursos educativos, discapacidad, inclusión.

\begin{abstract}
The Digital Educational Resources undoubtedly contribute to create inclusive, learning communities that promote appropriate learning environments primarily based on students' interactions since every rhythm and learning style is respected. This also contributes to innovate and lead the learning process towards Equality. The main objective of this research was to analyze the Digital Educational Resources suggested by the Ministry of Education of Chile in order to approach students with any type of disability from a mixed perspective in terms of technical areas, pedagogy, and accessibility. The results evidence basic flaws such as, unavailability of resources, pedagogical weaknesses; mainly focusing on educational necessities of permanent status, most of the resources are foreign ones, and most importantly, there is no classification of any educational resource in terms of level of excellence.
\end{abstract}

Keywords: Education, technologies, educational resources, disability, inclusion.

\footnotetext{
1. Magister en Informática Educativa. Especialidad Informática Educativa, Académica, Departamento de Educación. Uni versidad de Los Lagos, Chile; email: yortiz@ulagos.cl

2. Doctora en Educación, Académica, Departamento de Educación. Universidad de los Lagos, Chile; email: c.soto@ulagos.cl

3. Doctora en Educación, Académica, Departamento de Educación. Universidad de los Lagos, Chile; email: s.munoz@ulagos.cl
} 


\section{Introducción}

La informática educativa surge en Chile como una iniciativa pública en 1992 con un alcance de dos escuelas en Santiago y, posteriormente, en la Región de la Araucanía con cerca de cien establecimientos, coordinado por Pedro Hepp (Hepp, 1998). En 1995, comienza la expansión nacional, con ello se logró una cobertura de 5 mil 300 colegios a lo largo de todo el país, considerando Isla de Pascua y la Antártica Chilena. Al año 2007, el Gobierno de Chile, a través del Proyecto Enlaces del Ministerio de Educación de Chile (MINEDUC) $)^{4}$, comienza la implementación del Plan, Tecnologías para una Educación de Calidad (TEC), con el objetivo de incrementar el equipamiento tecnológico de los establecimientos y asegurar su uso pedagógico. El plan estaba destinado a los niveles de Educación Parvularia, Educación Básica y Educación Media de los establecimientos subvencionados, y con una inversión cercana a los 200 millones de dólares en infraestructura.

Actualmente este plan, promueve las siguientes estrategias de acción: equipamiento y asistencia técnica a los establecimientos educacionales; perfeccionamiento docente en uso pedagógico de las TIC; fomento, gestión y desarrollo de propuestas en informática educativa, que aporten al logro de mejores aprendizajes; desarrollo de propuestas pertinentes, en el ámbito rural; fomento, gestión y desarrollo de habilidades ciudadanas en el uso de las TIC (MINEDUC, 2018).
Desde esta perspectiva, Enlaces permite promover la igualdad de oportunidades de acceso para que todos los estudiantes logren un manejo básico de estas herramientas y puedan formarse de modo competente en el nuevo mundo digital. Finalmente, Enlaces apunta a dos preocupaciones centrales de la política educativa actual: la calidad y la equidad de la educación.

De lo anterior se desprende que, desde el Ministerio de Educación chileno, se han hecho importantes esfuerzos por introducir recursos tecnológicos en las aulas en todos los niveles, pero no se visualiza la incidencia que aquello tiene en los procesos de enseñanza y aprendizaje. Surge entonces, la necesidad de fomentar en la Formación Inicial Docente y permanente de los profesionales de la Educación, diferentes dimensiones de las TIC, su uso y frecuencia con que éstas son utilizadas (Cabero, 2014).

Diversos organismos a nivel mundial están investigando el fenómeno de la tecnología en los procesos educativos de niños, jóvenes y docentes; y cómo estas impactan tanto a nivel local como social. La finalidad es poder consensuar en estándares mínimos de quienes deben enseñar a estas generaciones para convivir con la era digital. Es así como UNESCO plantea los estándares de competencias TIC para los docentes del mundo (UNESCO, 2008).

4. Dentro del documento, las autoras también harán referencia a esta entidad a través de sus siglas. 
La UNESCO establece Estándares de Competencias en Tecnologías de Información y Comunicación para docentes desde el año 2008, los cuales se orientan a mejorar su práctica en todas las áreas de su desempeño profesional, combinando las competencias en TIC con innovaciones en: pedagogía, plan de estudios (currículo), organización escolar, uso de TIC para mejorar sus estrategias de enseñanza, cooperar con sus colegas y; en última instancia, poder convertirse en líderes, de la innovación dentro de sus respectivas instituciones. Un objetivo paralelo de esta investigación, junto con analizar los recursos educativos digitales, es aportar en alguna medida con orientaciones que permitan a los docentes definir cuál o cuáles de estos recursos son los más adecuados de acuerdo a sus propios intereses profesionales.

Es oportuno señalar que las tecnologías por su potencialidad de transformación son fuente de numerosos conflictos, relacionados con el mal uso de los mismos sobre todo en el ámbito de las relaciones sociales, por ejemplo, el ciberacoso, grooming, sexting, entre otros; pero sin duda realiza aportes en múltiples dimensiones. Con su destacada cualidad de intersticialidad han alcanzado todos los ámbitos de la vida actual, también al plano educativo (Mena y Marcos, 1994). Por consecuencia es importante que el docente como mediador y guía del proceso de enseñanza y aprendizaje de sus estudiantes, se capacite y tome como pilar de su trabajo paradigmas socioculturales, científicos y tecnológicos que permitan la participación y el razonamiento lógico, involucrando el uso de las TIC en el aula, para convertirlas en materiales de apoyo relevantes y no un distractor (Eurydice, 2001). Esto implica disponer de material validado para trabajar en forma segura con sus estudiantes.

Al Respecto Aranega y Domenech (2001) mencionan que las tecnologías de información y comunicación permiten que el profesorado sea más receptivo a los cambios en la metodología y en el rol docente: orientación y asesoramiento, dinamización de grupos, motivación de los estudiantes, diseño y gestión de entornos de aprendizaje, creación de recursos y evaluación formativa. Según estos autores, se puede decir que las tecnologías son herramientas que provocan cambios en el proceso de enseñanza y aprendizaje y que, poco a poco, rompen esquemas docentes tradicionalistas en el aula.

La capacidad y flexibilidad de las tecnologías de la información y las comunicaciones para la socialización y/o interacción, el aprendizaje y conocimiento destacan su función formativa "por cuanto apoyan la presentación de determinados contenidos, lo que puede ayudar a guiar, facilitar y organizar la acción didáctica; así como condicionan el tipo de aprendizaje a obtener, ya que pueden promover diferentes acciones mentales en los alumnos" (Cabero, 2000, p. 144).

Herrera (2003) realiza un análisis de las principales funciones que tienen las tecnologías en el aprendizaje donde distingue la provisión de estímulos sensoriales y la mediación cognitiva. 
Para el primer caso se basa en el modelo del procesamiento humano de la información dado por Castañeda (2003), de manera que ubica las tecnologías dentro de los estímulos del medio ambiente y concluye esta apreciación planteando como estas son utilizadas como medio de interacción y comunicación en actividades de aprendizaje. Su función es, en esencia, la provisión de estímulos sensoriales, entre ellos refuerza la idea de su estimulación en dos dimensiones: su capacidad atencional y su capacidad motivadora.

Luego de analizar la relevancia de las tecnologías en todo ámbito de la sociedad es innegable la estrecha relación entre tecnología y educación, la contribución que esta hace al desarrollo de ambientes de aprendizaje variados y cercanos a la juventud, que atiendan a los marcados intereses de los estudiantes de estar siempre conectados y variar sus focos de atención y participación. Esto justifica el análisis que se hace de los recursos propuestos por el Ministerio de Educación de Chile por un equipo de docentes de la Universidad de Los Lagos, Campus Osorno; desde una perspectiva mixta (cuanti y cualitativa) que permita ser un aporte consistente para relevar estos recursos como agentes que favorecen y potencian habilidades en estudiantes que asocian algún tipo de discapacidad.

En el ámbito de la inclusión educativa en Chile existen normativas que la regulan: La Ley 20.201 (Biblioteca del Congreso Nacional, 1998b) que modifica el dfl no 2, del año 1998, de educación, sobre subvenciones a estableci- mientos educacionales y otros cuerpos legales. Otra es el Decreto Supremo N 170/2010 del Ministerio de Educación (Biblioteca del Congreso Nacional, 1998a), que fija normas para determinar los alumnos con necesidades educativas especiales que serán beneficiarios de las subvenciones para educación especial. Posterior a esto se establecen orientaciones para la implementación del decreto $n^{\circ} 170$ en programas de integración escolar (MINEDUC, 2010). De esta forma se cuenta con un sustento sólido para amparar la inclusión de estudiantes que asocian algún tipo de discapacidad, a través de los denominados proyectos o programas de integración escolar.

\section{Metodología}

El objetivo del estudio fue analizar los recursos educativos digitales propuestos por el Ministerio de Educación, para estudiantes que asocian algún tipo de discapacidad. De ahí que la investigación siguió una metodología mixta, definida según Hernández, Fernández y Sampieri (2010), como un proceso de recolección, análisis y vinculación de datos cuantitativos y cualitativos en un mismo estudio o una serie de investigaciones para responder a un planteamiento del problema y es de tipo descriptiva “(...) la investigación descriptiva es aquella que busca especificar las propiedades, características, y los perfiles importantes de personas, de grupos, de comunidades o cualquier otro fenómeno que se someta a un análisis" (Danhke, 1989, p. 58). 
Se recabó información mediante el análisis de recursos educativos digitales propuestos por el MINEDUC, los cuales presentan una ficha denominada "Ficha de Descripción, Catálogo de Recursos Educativos Digitales" que fue analizada para determinar si cumple con los propósitos establecidos por la misma.

Se utilizaron y aplicaron diversos instrumentos que permitieron un análisis cuantitativo $\mathrm{y}$ cualitativo de la calidad de los recursos educativos digitales. Éstos son:

- Matriz de análisis cuantitativo, para conocer en detalle los recursos y sus características, respondiendo a preguntas de base como: ¿Cantidad de recursos tecnológicos disponibles y no disponibles?, ¿Tipo de recursos?, ¿nacionalidad?, entre otros aspectos cuantificables.

- Análisis Teórico de aspectos relevantes de considerar al momento de elaborar y utilizar un Recurso Educativo Digital (RED), en estudiantes que poseen necesidades educativas especiales, lo que derivó en la definición de dimensiones de análisis para la clasificación de los recursos.

- Pauta de evaluación para los recursos propuestos por el Ministerio, que contempló el propósito, objetivo y funcionalidad de aquellos recursos, la que fue validada por expertos, en los ámbitos de: educación, informática educativa, metodología, currículum, entre otros.
- Análisis de Ficha de identificación y orientación pedagógica propuesta por el Ministerio, para cada recurso expuesto en su plataforma.

La población para la investigación es la totalidad de recursos educativos digitales propuestos el Ministerio de Educación de Chile ${ }^{5}$ (38), para contribuir en el desarrollo de aprendizajes de estudiantes que asocian algún tipo de discapacidad sean estas transitorias o permanentes.

Definición necesidades educativas especiales de carácter permanente y transitorio (Decreto $N^{\circ} 83$ de 2015)

Necesidades educativas especiales ${ }^{6}$ de carácter permanente son aquellas barreras para aprender y participar, diagnosticadas por profesionales competentes que determinados estudiantes experimentan durante toda su escolaridad, y que demandan al sistema educacional la provisión de apoyos y recursos adicionales o extraordinarios para asegurar su aprendizaje escolar.

Por lo general, las NEE de carácter permanente se presentan asociadas a discapacidad visual, auditiva, disfasia, trastorno autista, discapacidad intelectual y discapacidad múltiple.

Necesidades educativas especiales de carácter transitorio son las dificultades de aprendizaje que experimentan los estudiantes en algún momento de su vida escolar, diagnosticada por profesionales competentes,

5. Esta información se encuentra con más detalle en: http://portales.mineduc.cl/index3.php?id_portal=20\&id_ seccion $=4813 \&$ \&id_contenido $=25803$

6. Entiéndase este término también por sus siglas NEE 
que demandan al sistema educacional; por una parte, la provisión de apoyos y recursos adicionales o extraordinarios por un determinado período de su escolarización, para asegurar el aprendizaje y la participación de estos en el proceso educativo, y por otra, el desarrollo de capacidades en el profesorado para dar respuestas educativas de calidad a los diferentes estilos de aprendizaje, ritmos, capacidades e intereses que presentan los estudiantes.

Las NEE de carácter transitorio pueden presentarse asociadas a dificultades de aprendizaje, trastornos específicos del lenguaje (TEL), déficit atencional y coeficiente intelectual limítrofe (MINEDUC, 2015).

\section{Dimensiones de análisis}

Se analizaron desde el espacio en línea habilitado desde el Ministerio de Educación, las siguientes dimensiones:

\begin{tabular}{|c|c|}
\hline Dimensión & $\begin{array}{c}\text { Descripción de la dimensión de } \\
\text { análisis }\end{array}$ \\
\hline Técnica & $\begin{array}{l}\text { Estándares de calidad técnica para } \\
\text { un buen funcionamiento }\end{array}$ \\
\hline Pedagógica & $\begin{array}{l}\text { Aspectos pedagógicos necesarios } \\
\text { para su utilización en el ámbito } \\
\text { educativo, contenido, pertinencia, } \\
\text { actualización, didáctica, entre otros. }\end{array}$ \\
\hline $\begin{array}{c}\text { Accesi- } \\
\text { bilidad } \\
\text { Universal }\end{array}$ & $\begin{array}{l}\text { Requerimientos para ser accesible un } \\
\text { recurso a la diversidad educativa. }\end{array}$ \\
\hline
\end{tabular}

Figura 1. Dimensiones abordadas en el análisis de cada uno de los recursos disponibles en el Portal del MINEDUC.

\section{Resultados}

Existen resultados de tipo cuantitativo y cualitativo, lo que permite una visión integral de los recursos tecnológicos. En la primera revisión al portal del Ministerio de Educación se evidenció que, del cien por ciento de recursos educativos digitales, el 79\% estaban disponibles, mientras que el $21 \%$ no lo estaban, debido a que no era posible su ingreso desde el enlace presentado.

Dentro del porcentaje de recursos disponibles, se pudo constatar que el $73 \%$ de ellos cumplían los requerimientos para ser evaluados y solo el $27 \%$ no los cumplían, debido a que eran programas utilitarios. Estos son definidos como medios auxiliares de programación, aplicaciones especiales o herramientas que sirven para facilitar las tareas del usuario y ayudarle a gestionar el ordenador.

Los programas utilitarios se centran en hacer que la ejecución de las tareas habituales resulte más sencilla e intuitiva. Algunos de ellos comienzan a incorporarse como rutina dentro de los sistemas operativos modernos. Son aplicaciones no tan grandes que realizan funciones muy específicas y concretas, según Melo y Alvarado (2014).

La modalidad de uso es un aspecto relevante si se considera la facilidad o dificultad de acceso a la información. La tabla 1 muestra la distribución, en la que dual es el recurso que permite el trabajo en ambas modalidades, ya sea descargable o en línea. 
Tabla 1. Modalidad de uso

\begin{tabular}{|c|c|}
\hline Tipo de recurso & Porcentaje \\
\hline Descargables & $73 \%$ \\
\hline En línea & $17 \%$ \\
\hline Dual & $10 \%$ \\
\hline
\end{tabular}

La mayor parte de los recursos no son chilenos (76.66\%), lo que evidencia la dependencia de materiales desarrollados en otras latitudes, pues tan solo el (23.33\%) son desarrollados en Chile.

Se indican en la tabla 2, las necesidades educativas especiales (NEE), a las cuales aportan los distintos recursos educativos digitales. Hay una distribución o cobertura importante en las distintas NEE que se pueden encontrar en los centros educativos.

En la tabla 3 se muestran los tipos de necesidades educativas especiales a los que van dirigidos: Necesidades educativas especiales permanentes (NEEP) y transitorias (NEET). Deja en evidencia una prevalencia hacia las NEEP sobre las NEET en específico, aunque hay recursos que responden a ambas, lo cual es positivo.
Tabla 2. Necesidades educativas especiales a las que van dirigidos los recursos

\begin{tabular}{|c|c|}
\hline Necesidad educativa especial & Porcentaje \\
\hline Discapacidad Auditiva & $12 \%$ \\
\hline Discapacidad Visual & $10 \%$ \\
\hline Deficiencias Múltiples & $11.1 \%$ \\
\hline Discapacidad Intelectual & $13.8 \%$ \\
\hline Discapacidad Motora & $6.5 \%$ \\
\hline $\begin{array}{c}\text { Trastornos del Espectro Autis- } \\
\text { ta (TEA) }\end{array}$ & $13.8 \%$ \\
\hline $\begin{array}{c}\text { Trastornos Específicos del } \\
\text { Lenguaje (TEL) }\end{array}$ & $6.5 \%$ \\
\hline $\begin{array}{c}\text { Trastorno por déficit de } \\
\text { atención con hiperactividad } \\
\text { (TDAH) }\end{array}$ & $13.8 \%$ \\
\hline $\begin{array}{c}\text { Dificultades Específicas del } \\
\text { Aprendizaje (DEA) }\end{array}$ & $12.5 \%$ \\
\hline \begin{tabular}{c} 
Total \\
\hline
\end{tabular} & $\mathbf{1 0 0 \%}$ \\
\hline
\end{tabular}

Tabla 3. Tipos de necesidades educativas

\begin{tabular}{|c|c|}
\hline $\begin{array}{c}\text { Tipo de Necesidad educativa } \\
\text { especial }\end{array}$ & Porcentaje \\
\hline NEE Permanentes (NEEP) & $60 \%$ \\
\hline NEE Transitorias (NEET) & $0 \%$ \\
\hline Ambas & $40 \%$ \\
\hline
\end{tabular}




\section{Evaluación delos Recursos Educativos Digitales}

Considerando aspectos técnicos, pedagógicos y de accesibilidad universal, se elaboró una pauta o rúbrica de evaluación asignando puntajes por segmentos de cumplimiento total, parcial o ausencia, divididos en:

- Excelente: Cumple con todos los estándares de calidad.

- Bueno: Cumple con la mayoría de los estándares de calidad.

- Regular: Cumple con algunos de los estándares de calidad.

- Malo: No cumple con los estándares mínimos de calidad.

De esta forma se buscó evaluar de la forma más objetiva y precisa posible, evitando así la incidencia de apreciaciones personales o subjetividad. El evaluar cada recurso permitió clasificarlos en términos de si cumplen los criterios presentes en la pauta elaborada, quedando en evidencia la heterogeneidad en cuanto a calidad.

Lo que es claro es que no se dispone de recursos a nivel de excelente o bueno en forma exclusiva dentro de la propuesta ministeria. (Ver tabla 4).

\section{Calidad Técnica}

El concepto técnico dice relación con asegurar a la calidad del producto desde ese punto de vista específicamente; pudiéndose realizar un análisis estructural de elementos tales
Tabla 4. Clasificación de los recursos educativos digitales disponibles y evaluables según puntaje de pauta de evaluación

\begin{tabular}{|c|c|}
\hline $\begin{array}{c}\text { Clasificación general de los } \\
\text { recursos }\end{array}$ & Cantidad \\
\hline Excelente & $0 \%$ \\
\hline Bueno & $50 \%$ \\
\hline Regular & $27 \%$ \\
\hline Malo & $23 \%$ \\
\hline
\end{tabular}

como el diseño (Cataldi, 2000). Esto implica un análisis de su funcionamiento integral como recurso tecnológico.

En esta dimensión, la mayoría de los recursos educativos digitales tienen características que cumplen con requisitos establecidos, según la pauta de evaluación, para que el uso, fiabilidad, distribución y el funcionamiento de los recursos sea óptimo.

Tabla 5. Evaluación calidad técnica

\begin{tabular}{|c|c|}
\hline $\begin{array}{c}\text { Clasificación según } \\
\text { evaluación técnica }\end{array}$ & Cantidad \\
\hline Excelente & $68 \%$ \\
\hline Bueno & $5 \%$ \\
\hline Regular & $18 \%$ \\
\hline Malo & $9 \%$ \\
\hline
\end{tabular}




\section{Calidad Pedagógica}

Dice relación con la utilidad pedagógica del recurso, el aporte al proceso de enseñanza y aprendizaje y el contenido que posee; aspectos prioritarios al momento de proponer algún recurso educativo digital al profesorado y/o público en general desde organismos ministeriales. Los resultados evidencian una deficiencia significativa. La clasificación se presenta en la tabla 6 .

Tabla 6. Evaluación Pedagógica

\begin{tabular}{|c|c|}
\hline $\begin{array}{c}\text { Clasificación según } \\
\text { evaluación pedagógica }\end{array}$ & Cantidad \\
\hline Excelente & $0 \%$ \\
\hline Bueno & $32 \%$ \\
\hline Regular & $23 \%$ \\
\hline Malo & $45 \%$ \\
\hline
\end{tabular}

En la tabla 7 se muestra la evaluación de la accesibilidad universal de los recursos, con acotaciones puntuales como: cumple con la condición de diseño para todos, puede ser utilizado en igualdad de condiciones por todas las personas; la instalación es sencilla y rápida, el diseño del recurso es útil y accesible para todas las personas. Luego del análisis se concluye que ningún recurso se puede categorizar como excelente; pero el $73 \%$ de ellos son catalogados como buenos, el $9 \%$ es considerado como regular y el $18 \%$ como malos.

El incorporar la evaluación de accesibilidad universal responde a un análisis de la relevancia de este aspecto, derivado de la inclusión y
Tabla 7. Evaluación accesibilidad universal

\begin{tabular}{|c|c|}
\hline $\begin{array}{c}\text { Clasificación según } \\
\text { accesibilidad universal }\end{array}$ & Porcentaje \\
\hline Excelente & $0 \%$ \\
\hline Bueno & $73 \%$ \\
\hline Regular & $9 \%$ \\
\hline Malo & $18 \%$ \\
\hline
\end{tabular}

la validación de este concepto a nivel nacional e internacional. Es así como la Real Academia Española $^{7}$ define la cualidad de accesible como "que tiene acceso", "de fácil acceso o trato" o "de fácil comprensión, inteligible”. Según el observatorio de la accesibilidad ${ }^{8}$, esta se puede definir como la cualidad de fácil acceso para que cualquier persona -incluso aquellas que tengan limitaciones en la movilidad, en la comunicación o el entendimiento- pueda llegar a un lugar, objeto o servicio. Esta concepción concuerda con la planteada por Fundación ONCE (2017), que señala que la accesibilidad es la condición que deben cumplir los entornos, procesos, bienes, productos y servicios, así como los objetos o instrumentos, herramientas y dispositivos, para ser comprensibles, utilizables y practicables por todas las personas en condiciones de seguridad y comodidad, y de la forma más autónoma y natural posible. Presupone la estrategia de "diseño para todos" y se entiende, sin perjuicio de los ajustes razonables, que deban adoptarse ${ }^{9}$.

7. Para mayor información, revisar el siguiente enlace: http://lema.rae.es/drae/?val=

8. Para mayor información, revisar el siguiente enlace: http://www.observatoriodelaaccesibilidad.es/accesibilidad/accesibilidad/definicion/

9. Para mayor información, revisar el siguiente enlace: http://rsed.fundaciononce.es/glosario.html 


\section{Discusión}

Disponer de recursos tecnológicos en internet es una gran ventaja para la educación, en esto se coincide con varios autores como Gutiérrez (2003), quien señala que "el desarrollo de Internet constituye el acontecimiento actual más influyente en la lectoescritura y la alfabetización”. Cabero (2007) indica que "la velocidad, el cambio y la transformación se han convertido en una de las características de nuestra sociedad" (p.177). Según Jordi Marín (2011) “(...) la sociedad actual es una sociedad extraordinariamente cambiante en todos los aspectos: económico, social, profesional, relacional...hemos pasado de la era industrial a la era de la información y del conocimiento con una rapidez sorprendente" (p.20).

Este escenario sitúa al sistema educativo como un espacio especialmente sensible a estos cambios sociales, en que los docentes son actores protagónicos al momento de definir los recursos educativos más idóneos para el trabajo con sus estudiantes, es así como Cabero (2004) nos recalca que

(...) tenemos que tener presente que en la actualidad el principal problema que se nos presenta no es el de acceder a la información...podemos decir que el problema no es la información, si no que hacer con tanta información, ¿cómo la filtramos?, ¿cómo sabemos que es pertinente, veraz? Es decir, el saber cribar la información, para quedarnos con lo que realmente nos interesa (p. 156).
Lo anterior avala procesos investigativos como el expuesto, ya que luego de describir, analizar y aplicar un proceso de evaluación sobre los recursos educativos digitales, se pudo dar respuesta a la interrogante sobre si estos recursos propuestos por el Ministerio de Educación de Chile cumplen criterios de calidad para el apoyo al aprendizaje de estudiantes que asocian algún tipo de discapacidad. Estos criterios se traducen en si cumplen criterios técnicos, pedagógicos y de accesibilidad. Martínez (2016) señala que las tecnologías de la información y la comunicación (TIC) aplicadas a la educación en general, y particularmente las tecnologías de apoyo a la diversidad (TAD), no deben convertirse en un elemento más de marginación y discriminación a nivel educativo, laboral o social, sino que deben ser elemento clave para la inclusión. Desde esta perspectiva, se toma como objetivo considerar las TAD como un recurso importante, a veces imprescindible, para el desarrollo integral y profesional de las personas con necesidades específicas de apoyo educativo (NEAE) (p.53).

El primer resultado de la investigación muestra que del $100 \%$ (38) de los recursos propuestos por el ministerio, el 79\% (30) estaban disponibles y 21\% (8) no disponibles. Esto significa que no existe en la web un proceso de actualización permanente de estos materiales, existiendo una clara deficiencia desde organismos que debiesen garantizar la actualización y vigencia de estos espacios en línea. Sin duda, el incorporar tecnologías para atender a la diversidad y lograr una inclusión efectiva es una gran responsabilidad, 
así lo recalca Martínez (2016) al señalar sobre "las tecnologías que su uso didáctico permite atender a la diversidad para ofrecer una respuesta real y más acorde con las exigencias y demandas actuales" (p.54).

Un aspecto positivo es que los recursos disponibles cubren todas las necesidades educativas especiales que están presentes en el denominado proyecto o programa de integración escolar (PIE), lo que permite al docente tener una variedad de posibilidades para abordar todas las áreas, cursos y niveles. Esto es una ventaja en el espacio analizado, lo que se condice con las oportunidades que las tecnologías entregan a estudiantes con necesidades específicas, entre ellas $\mathrm{Ca}$ bero (2008) menciona:

- Ayudan a superar las limitaciones que se derivan de las discapacidades cognitivas, sensoriales y motóricas del alumnado.

- Favorecen la autonomía de los estudiantes, pudiéndose adaptar a las necesidades y demandas de cada alumno de forma personalizada.

- Ofrecen un feed-back inmediato.

- Ahorran tiempo para la adquisición de habilidades y capacidades en los estudiantes.

- Favorecen el diagnóstico de los alumnos.

- Respaldan un modelo de comunicación y de formación multisensorial.

- Propician una formación individualizada, y el que los alumnos puedan avanzar a su propio ritmo, lo cual es de extremada importancia para los sujetos con algún tipo de discapacidad.

- Favorecen el desarrollo de la autonomía e independencia de las personas.

- Evitan la marginación, la brecha digital, que introduce el verse desprovisto de utilizar las herramientas de desarrollo de la sociedad del conocimiento.

- Facilitan la inserción sociolaboral de aquel alumnado con dificultades específicas.

Ahondando en lo anterior, se evidencia que la mayoría de estos recursos están dirigidos a necesidades educativas especiales de tipo permanente. Según el Decreto No 170 en un Proyecto o Programa de Integración Escolar, hay más disponibilidad para participar de este programa para niños con necesidades educativas especiales de tipo transitorias; por esta razón sería necesario que los recursos propuestos por el Ministerio de Educación de Chile fuesen dirigidos de manera equitativa para necesidades educativas permanente y transitorias, debido a que las transitorias son las necesidades que predominan en un programa de integración escolar y las permanentes son las que precisan más herramientas de apoyo.

Los recursos tienen una adecuada calidad técnica, pero para que un recurso sea pertinente en el ámbito educativo debe responder a ciertas condiciones para el uso pedagógico, y la mayoría no cumple con esta exigencia; por lo tanto, se necesita de una revisión ge- 
neral por parte del organismo que los publica para certificar su uso en el ámbito educativo.

Esto se traduce en considerar criterios de calidad acordes a las exigencias para participar en forma constructiva en educación; asimismo, Burbules (2001) nos indica que "lo que los usuarios encuentran en la Internet es también en sí mismo un factor que influye en quiénes participan, cómo y cuánto. Estos se convierten también en criterios tácitos de inclusión y exclusión" (p.51).Desde el plano de accesibilidad universal se puede concluir que los recursos son accesibles para la gran mayoría de las personas, centrados más que nada en los aspectos básicos de este concepto, como utilizables y practicables por los usua- rios; en condiciones de seguridad y comodidad, de la forma más autónoma y natural posible. Martínez (2016) señala que

(...) una tecnología es accesible cuando la puede usar cualquier persona. Es una condición necesaria para la participación social de todos y garantía de un mejor diseño para todos. En una sociedad en la que cada vez se utilizan más las TIC, asegurar la accesibilidad de los medios tecnológicos y de los contenidos resulta prioritario (p.56).

\section{Referencias}

Aranega, S. y Domenech, J. (2001). La educación primaria: retos, propuestas y dilemas. Barcelona: Graó

Biblioteca del Congreso Nacional de Chile (1998a). Biblioteca del Congreso Nacional de Chile - Decreto 170 del 2010

Biblioteca del Congreso Nacional de Chile (1998b). Biblioteca del Congreso Nacional de Chile - Ley 20.201 Modifica el DFL no 2, de 1998, de Educacion, sobre subvenciones a establecimientos educacionales y otros cuerpos legales, 4-7

Burbules, N., y Callister, T. (2001). Las promesas de riesgo y los riesgos promisorios de las nuevas tecnologías de la información en educación. Educación-Riesgos y promesas de las nuevas tecnologías de la Información. España

Cabero, J. (2000). Las nuevas tecnologías de la información y comunicación: aportaciones a la enseñanza. Nuevas tecnologías aplicadas a la educación. Madrid

Cabero, J. (2008). TIC para la igualdad: la brecha digital en la discapacidad. Revista Anales, 8 (2), 21 - 22. Recuperado de http://goo.gl/LRqNdD 
Cabero, J. (2014). Formación del profesorado universitario en TIC. Aplicación del método Delphi para la selección de los contenidos formativos. Educación XXI, 17 (1), 111 - 132

Cabero, J.; Martínez, F. y Prendes, M. P. (2007). Profesor, ¿estamos en el ciberespacio? Davinci, La Coruña

Cabero, J. y Romero, R. (2004). Nuevas tecnologías en la práctica educativa. Granada. ARIAL

Careaga, M. y Avendaño, A. (2007). Estándares y Competencias TIC para la formación inicial de profesores. Revista de Estudios y Experiencias en Educación (12), 93-106

Castañeda, H, Á.E. (2003). El papel de las tecnologías de la información y las comunicaciones (TICs) en el proceso de enseñanza aprendizaje a comienzos del siglo XXI. In: M.E. De la Vega García (Editor), Preparación pedagógica integral para profesores universitarios, Habana

Cataldi, Z. (2000). Una metodología para el diseño, desarrollo y evaluación de software educativo (Doctoral dissertation, Facultad de Informática)

Danhke, G.L (1989). Investigación y comunicación. En C. Fernández-Collado y G.L Danhke (Comps.). La comunicación humana: Ciencia social. México, D.F.: McGraw-Hill de México, 385-454

Fundación Once (2017). Guía de responsabilidad social empresarial y discapacidad de la Fundación ONCE. Recuperado de http://rsed.fundaciononce.es/glosario.html

Gutiérrez M., A. (2003). Alfabetización digital. Algo más que ratones y teclas. Barcelona: Gedisa

Hepp, P. (1998). Experiencias chilenas con la informática educativa. La educación en la era de la informática: qué da resultado y qué no, 123-133

Hernández, R.; Fernández, C. y Baptista, P. (2010). Metodología de la Investigación. $5^{\circ}$ edición. México: McGraw Hill Interamericana

Herrera, M. y Castón, P. (2003). Las políticas sociales en las sociedades complejas. Ariel, Barcelona

Marcos, M. y Mena, B. (1994). Nuevas tecnologías para la enseñanza. Didáctica y la Metodología. Madrid: Editorial de la Torre

Martín, G. (2003). Alfabetización digital: algo más que ratones y teclas. Gedisa 
Martínez, M. E. (2016). Atención a la diversidad con tecnologías. En Gallego-Arrufat, M. J. y Raposo-Rivas, M. (Coord.). Formación para la educación con tecnologías, p. 53. Madrid: Ediciones Pirámide

Melo, O. y Alvarado, N. (2014) Software utilitario. Recuperado de https://prezi.com/_ immmt347_pd/software-utilitario/

Ministerio de Educación de Chile (2010). Orientaciones Para La Implementación Del Decreto No 170 En Programas De Integración Escolar, 1-16

Ministerio de Educación de Chile (2015). Diversificación de la enseñanza Decreto Nº3/2015, 41. Recuperado de http://portales.mineduc.cl/usuarios/edu.especial/File/2015/Decreto 83-2015.pdf

Ministerio de Educación de Chile (2017). Recursos Educativos Digitales. Recuperado de http://portales.mineduc.cl/index3.php?id_portal=20\&id_seccion=4813\&id_contenido $=25803$

Ministerio de Educación de Chile (2017). Educación Especial. Gobierno de chile (en línea). Recuperado de http://www.educacionespecial.mineduc.cl/

Ministerio de Educación de Chile (2018). Tecnologías. Recuperado de https://www.mineduc. $\mathrm{cl} /$ ?s=tecnolog $\% \mathrm{C} 3 \% \mathrm{ADas}$

Ministerio de Educación de España, Cultura y Deporte (2001). Indicadores básicos de la incorporación de las TIC a los sistemas educativos europeos. Eurydice. Recuperado de http://books.google.es/books?id=tsRiwyhnq7wC\&printsec=frontcover\&hl=es\&source =gbs_ge_summary_r\&cad $=0 \# \mathrm{v}=$ onepage $\& \mathrm{q} \& \mathrm{f}=$ false

Monfort, J. M. y Aspachs, R. B. (2011). Enseñar en la sociedad del conocimiento: reflexiones desde el pupitre. ICE Universitat de Barcelona

Organización de las Naciones Unidas (2008). Estándares de competencias en tic para docentes. Recuperado de http://www. eduteka. org/EstandaresDocentesUnesco. php

Prensky, M. (2010). Nativos e inmigrantes digitales. Distribuidora SEK

Real Academia española (2016). Diccionario de la Lengua Española. Recuperado de: http://dle. rae.es/?id=SkrC5M4 